\title{
A CONGRUENCE THEOREM FOR CLOSED HYPERSURFACES IN RIEMANN SPACES
}

\author{
HEINZ BRÜHLMANN
}

\section{Introduction}

We consider two closed oriented surfaces $F$ and $\bar{F}$ in Euclidean 3 -space $E^{3}$ and a differentiable map $\Phi: F \rightarrow \bar{F}$ preserving the orientation. The word differentiable always means differentiable of class $C^{\infty}$. Furthermore, we assume that the set of points on $F$ where the lines $(p, \bar{p}), \bar{p}=\Phi(p)$, are tangent to $F$ does not have inner points. Then the following theorems are known:

A) If all the lines $(p, \bar{p})$ are parallel and $H(p)=\bar{H}(\bar{p})(H$ and $\bar{H}$ are the mean curvatures of $F$ and $\bar{F}$ respectively), then the surface $\bar{F}$ is obtained from $F$ by a single translation, i.e., the distances $p \bar{p}$ are the same for all points $p$ on $F$ (H. Hopf and K. Voss [8]).

B) If all the lines $(p, \bar{p})$ go through a fixed point 0 (which does or does not lie on $F$ or $\bar{F}$ ) and if $r H(p)=\bar{r} \bar{H}(\bar{p})$ ( $r$ and $\bar{r}$ are the distances of $p$ and $\bar{p}$ from 0 ), then $F$ is obtained from $F$ by a homothety, in other words the ratio $\bar{r} / r$ is constant (A. Aeppli [1]).

In order to generalize these two theorems we consider the following case: Let $R^{n+1}$ be an $(n+1)$-dimensional Riemann space, and $\Phi(p, s)$ be a oneparameter group of transformations of $R^{n+1}$ into itself. Furthermore, let $F^{n}$ and $\bar{F}^{n}$ be two $n$-dimensional hypersurfaces of $R^{n+1}$ such that the points of $\bar{F}^{n}$ are given by the formula:

$$
\bar{p}=\Phi(p, f(p)), \quad p \in F^{n},
$$

where $f(p)$ is a differentiable function of $F^{n}$. To generalize the condition for the mean curvatures, we have to introduce an additional family of hypersurfaces, one for every point of $F^{n}$, given by the formula:

$$
\tilde{F}_{p}^{n}=\Phi\left(F^{n}, f(p)\right) .
$$

Then the point $\bar{p}=\Phi(p, f(p))$ lies on the hypersurfaces $\bar{F}^{n}$ and $\tilde{F}_{p}^{n}$ and we define:

$$
\begin{aligned}
& \bar{H}(\bar{p})=\text { mean curvature of } \bar{F}^{n} \text { at } \bar{p}, \\
& \tilde{H}(\bar{p})=\text { mean curvature of } \tilde{F}_{p}^{n} \text { at } \bar{p} .
\end{aligned}
$$

Received June 5, 1972, and, in revised form, July 12, 1973. 
We denote by $S$ the set of points of $\bar{F}^{n}$ where the vector tangent to the orbit of $\Phi(p, s)$ through $\bar{p}$ lies in the tangent space of $\bar{F}^{n}$. For this general case, the following two theorems are known.

I) If $\bar{H}(\bar{p})=\tilde{H}(\bar{p})$ for all $\bar{p} \in \bar{F}^{n}, \Phi(p, s)$ is a group of homothetic transformations, and the set $S$ of the exceptional points is nowhere dense in $\bar{F}^{n}$, then $F^{n}$ and $\bar{F}^{n}$ are congruent $\bmod \Phi$; in other words, $f(p)=$ const. (Y. Katsurada [9]).

II) If $\bar{H}(\bar{p})=\tilde{H}(\bar{p})$ for all $\bar{p} \in \bar{F}^{n}$, and the set $S$ is empty, then $F^{n}$ and $\bar{F}^{n}$ are congruent mod $\Phi(\mathrm{H}$. Hopf and Y. Katsurada [7]).

Theorem II is not a generalization of Theorem A, since in this case we always have exceptional points. However it suggests that Theorem $I$ is true without the additional assumption of homotheticity.

Theorem I has been proved by Y. Katsurada by using the method of differential forms. For the proof of Theorem II the authors use the strong maximum principle of $E$. Hopf [5]. In [10], K. Voss gave a proof of Theorem A, using a generalized maximum principle. However, his proof worked only in the case where $F$ and $\bar{F}$ are real analytic surfaces. Later, P. Hartman [3] gave a proof without using the assumption of analyticity, by generalizing the strong maximum principle for elliptic differential equations. In this paper we give a proof of the following theorem, which is a generalization of Theorem II since we may have exceptional points, but which is not a generalization of Theorem I since the assumption on the exceptional points is stronger than that in Theorem I.

Theorem. Let $F^{n}, \bar{F}^{n}, \tilde{F}_{p}^{n}$ be closed oriented hypersurfaces in $R^{n+1}$ as explained above, and assume all maps to be orientation-preserving. Furthermore let $\varphi(\bar{p})=(w, \bar{n})$, where $w$ is the vector tangent to the curve $\Phi(\bar{p}, s),-\varepsilon<$ $s<+\varepsilon$, at $\bar{p}$, and $\bar{n}$ is the normal vector of $\bar{F}^{n}$ at $p$. If $\operatorname{grad} \varphi \neq 0$ whenever $\varphi=0$ on $\bar{F}^{n}$, and $\bar{H}(\bar{p})=\tilde{H}(\bar{p})$ for all $\bar{p} \in \bar{F}^{n}$, then the hypersurfaces $F^{n}$ and $\bar{F}^{n}$ are congruent $\bmod \Phi$.

\section{Variation of the mean curvature}

Let $F^{n}$ be a hypersurface in an $(n+1)$-dimensional Riemann space $R^{n+1}$ given locally by the equations

$$
x^{i}=x^{i}\left(u^{\alpha}\right), \quad i=1, \cdots, n+1 ; \quad \alpha=1, \cdots, n .
$$

Then the tangent space to the surface is spanned by the $n$ linearly independent vectors $t_{\alpha}=\left(\partial x^{i} / \partial u^{\alpha}\right) \partial / \partial x^{i}$. For the covariant derivative of the vector-field $t_{\alpha}$ in the direction of $t_{\beta}$ in $R^{n+1}$ we get

$$
D_{\beta} t_{\alpha}=\nabla_{t_{\beta}} t_{\alpha}=\left(\frac{\partial^{2} x^{i}}{\partial u^{\alpha} \partial u^{\beta}}+\Gamma_{j k}^{i} \frac{\partial x^{j}}{\partial u^{\alpha}} \frac{\partial x^{k}}{\partial u^{\beta}}\right) \frac{\partial}{\partial x^{i}},
$$

and for the second fundamental form 


$$
l_{\alpha \beta}=\left(D_{\alpha} t_{\beta}, n\right)=g_{i l}\left(\frac{\partial^{2} x^{i}}{\partial u^{\alpha} \partial u^{\beta}}+\Gamma_{j k}^{i} \frac{\partial x^{j}}{\partial u^{\alpha}} \frac{\partial x^{k}}{\partial u^{\beta}}\right) n^{l},
$$

where $n=n^{i} \partial / \partial x^{i}$ is the normal to the hypersurface, and $g_{i j}$ is the metric tensor of the space $R^{n+1}$. The formula for the mean curvature of the hypersurface is

$$
H=g^{\alpha \beta} l_{\alpha \beta} / n=l_{\alpha}^{\alpha} / n,
$$

where $g^{\alpha \beta}$ is the inverse of $g_{\alpha \beta}=g_{i j}\left(\partial x^{i} / \partial u^{\alpha}\right) \partial x^{j} / \partial u^{\beta}$.

Now let $\Phi(p, s)$ be a one-parameter group of transformations of $R^{n+1}$, and $F^{n}$ and $\bar{F}^{n}$ be two hypersurfaces such that

$$
\bar{F}^{n}=\left\{\Phi(p, f(p)), p \in F^{n}\right\}
$$

as in the introduction. We introduce an additional family of hypersurfaces, depending on a point $p \in F^{n}$ and a parameter $t, 0 \leq t \leq 1$, given by the equation

$$
F^{n}(p, t)=\left\{\Phi(q, t f(q)+(1-t) f(p)) \mid q \in F^{n}\right\} .
$$

Since $\Phi(p, t f(p)+(1-t) f(p))=\Phi(p, f(p))=\bar{p}$, the point $\bar{p}$ lies on all the hypersurfaces $F^{n}(p, t), p$ fixed and $0 \leq t \leq 1$. Furthermore we have, for $t=1$,

$$
F^{n}(p, 1)=\left\{\Phi(q, f(q)) \mid q \in F^{n}\right\}=\bar{F}^{n},
$$

and, for $t=0$,

$$
F(p, 0)=\left\{\Phi(q, f(p)) \mid q \in F^{n}\right\}=\tilde{F}_{p}^{n} .
$$

From these relations we get

$$
\bar{H}(\bar{p})-\tilde{H}(\bar{p})=\int_{0}^{1} \frac{d H(p, t)}{d t} d t,
$$

where $H(p, t)$ is the mean curvature of $F^{n}(p, t)$ at the point $\vec{p}$.

The variation of the mean curvature gives

$$
d H(p, t) / d t=l_{\alpha \beta} d g^{\alpha \beta} / d t+g^{\alpha \beta} d l_{\alpha \beta} / d t,
$$

and by differentiating the relation $g^{\alpha \beta} g_{\gamma \beta}=\delta_{\gamma}^{\alpha}$ we get

$$
d g^{\alpha \beta} / d t=-g^{\alpha \delta} g^{\beta r} d g_{\gamma^{\delta}} / d t=-g^{\alpha \grave{\delta}} g^{\beta \gamma}\left\{\left(d t_{\gamma} / d t, t_{\grave{\delta}}\right)+\left(t_{\gamma}, d t_{\tilde{\delta}} / d t\right)\right\} .
$$

Furthermore, by taking the covariant derivative of the relations $(n, n)=1$ and $\left(n, t_{\alpha}\right)=0$, we obtain $\left(n, D_{\alpha} n\right)=0$ and $\left(D_{\alpha} n, t_{\beta}\right)+\left(n, D_{\alpha} t_{\beta}\right)=0$ or $D_{\alpha} n$ $=\lambda_{\alpha}^{\beta} t_{\beta}$ with $\lambda_{\alpha}^{\beta}=-\left(n, D_{\alpha} t_{r}\right) g^{\gamma^{\beta}}$. Hence

$$
D_{\alpha} n=-\left(n, D_{\alpha} t_{\gamma}\right) g^{\beta \gamma} t_{\beta},
$$




$$
\frac{d g^{\alpha \beta}}{d t} l_{\alpha \beta}=-g^{\alpha \delta} g^{\beta \gamma}\left\{\left(\frac{d t_{r}}{d t}, t_{\delta}\right)+\left(t_{\gamma}, \frac{d t_{\delta}}{d t}\right)\right\} \quad\left(D_{\alpha} t_{\beta}, n\right)=2 g^{\alpha \beta}\left(D_{\alpha} n, t_{\beta}\right) .
$$

In order to compute the second term in the above expression for $d H(p, t) / d t$, differentiating the relations $(n, n)=1$ and $\left(n, t_{\alpha}\right)=0$ with respect to $t$ we get $(d n / d t, n)=0$ and $\left(d n / d t, t_{\alpha}\right)+\left(n, d t_{\alpha} / d t\right)=0$, or $d n / d t=\lambda^{\alpha} t_{\alpha}$ with $\lambda^{\alpha}=$ $-\left(d t_{\beta} / d t, n\right) g^{\alpha \beta}$. Hence

$$
\begin{aligned}
d n / d t & =-\left(d t_{\beta} / d t, n\right) g^{\alpha \beta} t_{\alpha}, \\
g^{\alpha \beta} \frac{d l_{\alpha \beta}}{d t}=g^{\alpha \beta} \frac{d}{d t}\left(D_{\alpha} t_{\beta}, n\right) & =g^{\alpha \beta}\left(\frac{d}{d t} D_{\alpha} t_{\beta}, n\right)-g^{\alpha \beta} \Gamma_{\alpha \beta}^{\delta}\left(\frac{d t_{\delta}}{d t}, n\right),
\end{aligned}
$$

where $\Gamma_{\alpha \beta}^{\delta}=\left(D_{\alpha} t_{\beta}, t_{\gamma}\right) g^{\gamma \delta}$. Finally we get the following formula for the variation of the mean curvature:

$$
\frac{d H}{d t}=g^{\alpha \beta}\left(\frac{d}{d t} D_{\alpha} t_{\beta}, n\right)+2 g^{\alpha \beta}\left(D_{\alpha} n, \frac{d t_{\beta}}{d t}\right)-g^{\alpha \beta} \Gamma_{\alpha \beta}^{\delta}\left(\frac{d t_{\delta}}{d t}, n\right) .
$$

Now using the definition of the hypersurfaces $F^{n}(p, t)$ :

$$
F^{n}(p, t)=\left\{\Phi(q, t f(q)+(1-t) f(p)) \mid q \in F^{n}\right\},
$$

or in local coordinates

$$
x_{p}^{i}\left(u^{\alpha}, t\right)=\Phi^{i}\left(u^{\alpha}, t f\left(u^{\alpha}\right)+(1-t) f(p)\right),
$$

where $f(p)$ is independent of the $u^{\alpha}$, we get

$$
x_{\alpha}^{i}=\partial x_{p}^{i} / \partial u^{\alpha}=\partial \Phi^{i} / \partial u^{\alpha}+t\left(\partial \Phi^{i} / \partial s\right) \partial f / \partial u^{\alpha},
$$

so that for the tangent vectors $t_{\alpha}$ of the hypersurface $F^{n}(p, t)$ at the point $\bar{p}$ we have

$$
t_{\alpha}=\left(\partial \Phi^{i} /\left.\partial u^{\alpha}\right|_{\bar{p}}+w^{i} t \partial f / \partial u^{\alpha}\right) \partial / \partial x^{i},
$$

where $w^{i}=\partial \Phi(\bar{p}, s) /\left.\partial s\right|_{s=0}$, and by differentiating with respect to $t$

$$
d t_{\alpha} / d t=w \partial f / \partial u^{\alpha}, \quad w=w^{i} \partial / \partial x^{i} .
$$

Furthermore

$$
D_{\alpha} t_{\beta}=\left(\partial x_{\alpha}^{i} / \partial u^{\beta}+\Gamma_{j k}^{i} x_{\alpha}^{j} x_{\beta}^{k}\right) \partial / \partial x^{i}
$$

so

$$
\frac{d}{d t} D_{\alpha} t_{\beta}=\left(\frac{d}{d t} \frac{\partial x_{\alpha}^{i}}{\partial u^{\beta}}+\Gamma_{j k}^{i} \frac{\partial x_{\alpha}^{j}}{\partial t} x_{\beta}^{k}+\Gamma_{j k}^{i} x_{\alpha}^{j} \frac{\partial x_{\beta}^{k}}{\partial t}\right) \frac{\partial}{\partial x^{i}}
$$


For the derivative of $x_{\alpha}^{i}$ we get

$$
\begin{aligned}
\frac{\partial x_{\alpha}^{i}}{\partial u^{\beta}}= & \frac{\partial^{2} \Phi^{i}}{\partial u^{\alpha} \partial u^{\beta}}+t \frac{\partial^{2} \Phi^{i}}{\partial u^{\alpha} \partial s} \frac{\partial f}{\partial u^{\beta}}+t \frac{\partial^{2} \Phi^{i}}{\partial u^{\beta} \partial s} \frac{\partial f}{\partial u^{\alpha}} \\
& +t^{2} \frac{\partial^{2} \Phi^{i}}{\partial s^{2}} \frac{\partial f}{\partial u^{\alpha}} \frac{\partial f}{\partial u^{\beta}}+t \frac{\partial \Phi^{i}}{\partial s} \frac{\partial^{2} f}{\partial u^{\alpha} \partial u^{\beta}} .
\end{aligned}
$$

Since $\partial^{2} \Phi^{i} / \partial u^{\alpha} \partial u^{\beta}, \partial^{2} \Phi^{i} / \partial u^{\alpha} \partial s, \partial^{2} \Phi^{i} / \partial s^{2}, \partial \Phi^{i} / \partial s$ do not depend on $t$ when considered only at the point $\bar{p}$ on the hypersurfaces $F^{n}(p, t)$, we get for the derivative of the above expression with respect to $t$ :

$$
\frac{d}{d t} \frac{\partial x_{\alpha}^{i}}{\partial u^{\beta}}=\frac{\partial w^{i}}{\partial u^{\alpha}} \frac{\partial f}{\partial u^{\beta}}+\frac{\partial w^{i}}{\partial u^{\beta}} \frac{\partial f}{\partial u^{\alpha}}+2 t \frac{\partial w^{i}}{\partial s} \frac{\partial f}{\partial u^{\alpha}} \frac{\partial f}{\partial u^{\beta}}+w^{i} \frac{\partial^{2} f}{\partial u^{\alpha} \partial u^{\beta}},
$$

so

$$
\frac{d}{d t} D_{\alpha} t_{\beta}=w \frac{\partial^{2} f}{\partial u^{\alpha} \partial u^{\beta}}+D_{\beta} w \frac{\partial f}{\partial u^{\alpha}}+D_{\alpha} w \frac{\partial f}{\partial u_{\beta}}+2 t \frac{\partial w}{\partial s} \frac{\partial f}{\partial u^{\alpha}} \frac{\partial f}{\partial u^{\beta}}
$$

Therefore the formula for the variation of the mean curvature in our case is the following:

$$
\begin{aligned}
\frac{d H}{d t}= & (w, n) g^{\alpha \beta} \frac{\partial^{2} f}{\partial u^{\alpha} \partial u^{\beta}}+2 g^{\alpha \beta} \frac{\partial(w, n)}{\partial u^{\alpha}} \frac{\partial f}{\partial u^{\beta}}+2 t\left(\frac{\partial w}{\partial s}, n\right) g^{\alpha \beta} \frac{\partial f}{\partial u^{\alpha}} \frac{\partial f}{\partial u^{\beta}} \\
& -g^{\alpha \delta} \Gamma^{\beta}{ }_{\alpha \delta}(w, n) \frac{\partial f}{\partial u^{\beta}} .
\end{aligned}
$$

\section{A lemma on partial differential equations}

For the proof of our main theorem we need a generalization of the strong maximum principle for elliptic partial differential equations. We consider a linear differential expression of the form

$$
L(f)=\sum_{\alpha, \beta=1}^{n} A_{\alpha \beta}(x) \frac{\partial^{2} f}{\partial x^{\alpha} \partial x^{\beta}}+\sum_{\alpha=1}^{n} B_{\alpha}(x) \frac{\partial f}{\partial x^{\alpha}},
$$

where $A_{\alpha \beta}(x)$ and $B_{\alpha}(x)$ are differentiable functions, together with a differentiable function $\varphi(x)$ in a normal domain $G$ of the $n$-dimensional number space $\boldsymbol{R}^{n}$. We assume that $\varphi(x)$ and $L(f)$ have the following properties:

a) $\operatorname{grad} \varphi(x) \neq 0$ whenever $\varphi(x)=0$,

b) $\sum_{\alpha, \beta=1}^{n} A_{\alpha \beta}(x) \lambda^{\alpha} \lambda^{\beta}$ is positive definite for every $x$ with $\varphi(x)>0$, negative definite for every $x$ with $\varphi(x)<0$, and identically 0 for every $x$ with $\varphi(x)=0$.

Then we prove the following

Lemma. Let $f(x)$ be a solution of $L(f)=0$, and $x_{0}$ be a point in $G$ such 
that $f(x) \leq f\left(x_{0}\right)$ for all $x$ in $G$. If either $\varphi\left(x_{0}\right) \neq 0$ or $\varphi\left(x_{0}\right)=0$ and $\sum_{\alpha=1}^{n} B_{\alpha}\left(x_{0}\right)\left(\partial \varphi / \partial x^{\alpha}\right)\left(x_{0}\right)>0$, then $f(x) \equiv f\left(x_{0}\right)$ in a neighborhood of $x_{0}$.

This lemma is a special case of a theorem proved in the paper [4] by Hartman and Sacksteder. However, since we use only a simple case, we give a sketch of the proof.

Proof. The case $\varphi\left(x_{0}\right) \neq 0$ follows directly from the strong maximum principle of E. Hopf [5]. Therefore we may assume $\varphi\left(x_{0}\right)=0$, and $\sum_{\alpha=1}^{n} B_{\alpha}\left(\partial \varphi / \partial x^{\alpha}\right)\left(x_{0}\right)>0$. The proof for this case is a modification of the proof of E. Hopf's second lemma [6]. Since by assumption $\operatorname{grad} \varphi \neq 0$ whenever $\varphi=0$, the set of points where $\varphi(x)=0$ is a differentiable curve through $x_{0}$. Therefore there exists an open ball $K_{1}$ in $G$ such that its boundary has exactly the point $x_{0}$ in common with the curve $\varphi(x)=0$ and that $\varphi(x)>0$ in $\bar{K}_{1}-x_{0}$. We choose its center as the origin of the coordinate system, and set $r=|x|, r_{0}$ $=\left|x_{0}\right|$. We may assume $f\left(x_{0}\right)=0$ and $f(x) \geq 0$ in $K_{1}$. By the strong maximum principle this implies either $f(x)>0$ in $\bar{K}_{1}-x_{0}$ and $f\left(x_{0}\right)=0$ or $f(x) \equiv 0$ in $\bar{K}_{1}$. We show that $f(x)>0$ in $\bar{K}_{1}-x_{0}$ leads to a contradiction. We consider the auxiliary function $h(x)=e^{-r^{2}}-e^{r^{2}}$, which has the properties: $h(x)>0$ for $|x|<r_{0}, h(x)=0$ for $|x|=r_{0}$, and

$$
L(h)\left(x_{0}\right)=\sum_{\alpha=1}^{n} B_{\alpha} \frac{\partial h}{\partial x^{\alpha}}\left(x_{0}\right)=-2 e^{-r^{2}} \sum_{\alpha=1}^{n} B_{\alpha} x_{0}^{\alpha}=c \sum_{\alpha=1}^{n} B_{\alpha} \frac{\partial \varphi}{\partial x^{\alpha}}\left(x_{0}\right), \quad c>0,
$$

since the vector $x_{0}=\left(x_{0}^{1}, \cdots, x_{0}^{n}\right)$ is a negative multiple of $\operatorname{grad} \varphi$. Therefore $L(h)\left(x_{0}\right)>0$, and hence $L(h)>0$ in the closure of a ball $K_{2}$ with center $x_{0}$. Now we consider the function $g(x)=f(x)-\varepsilon h(x)$ in the domain $K=K_{1} \cap K_{2}$. Then $g \geq 0$ on $S_{1} \cap \bar{K}_{2}$, where $S_{1}=$ boundary of $K_{1}$, and $g\left(x_{0}\right)=0$. Furthermore, by choosing $\varepsilon>0$ sufficiently small, we also have $g \geq 0$ on $S_{2} \cap \bar{K}_{1}$, since $f>0$ there.

Since $L(f)=0$, and $L(h)>0$ in $K$, we have $L(g)<0$ in $K$, and therefore $g \geq 0$ in $K$ by the strong maximum principle. Hence $(d g / d n)\left(x_{0}\right) \leq 0$, where $d g / d n$ is the derivative in the direction of the outer normal of $K$. But then

$$
\frac{d f}{d n}\left(x_{0}\right)=\frac{d g}{d n}\left(x_{0}\right)+\varepsilon \frac{d h}{d n}\left(x_{0}\right)<0,
$$

since $(d h / d n)\left(x_{0}\right)<0$. This contradicts the fact that $\operatorname{grad} f\left(x_{0}\right)=0$.

Proof of the Theorem. By using the formula for the variation of the mean curvature and the relation

$$
\bar{H}(\bar{p})-\tilde{H}(\bar{p})=\int_{0}^{1} \frac{d H(p, t)}{d t} d t=0,
$$

we get the following differential equation for the function $f$ : 


$$
\sum_{\alpha, \beta=1}^{n} A_{\alpha \beta} \frac{\partial^{2} f}{\partial u^{\alpha} \partial u^{\beta}}+\sum_{\beta=1}^{n} B_{\beta} \frac{\partial f}{\partial u^{\beta}}=0
$$

where

$$
\begin{gathered}
A_{\alpha \beta}=\int_{0}^{1}(w, n(t)) g^{\alpha \beta}(t) d t \\
B_{\beta}=\int_{0}^{1}\left\{2 g^{\alpha \beta} \frac{\partial(w, n)}{\partial u^{\alpha}}+2 t\left(\frac{\partial w}{\partial s}, n\right) g^{\alpha \beta} \frac{\partial f}{\partial u^{\alpha}}-g^{\alpha \beta} \Gamma_{\alpha \delta}^{\beta}(w, n)\right\} d t
\end{gathered}
$$

From the relation

$$
(w, n(t)) d A(t)=(w, \bar{n}) d \bar{A}
$$

(proved in [2]), where $d A(t)$ is the volume element of the hypersurface $F^{n}(p, t)$ at $p$, it follows that if $(w, \bar{n}) \neq 0$, then $(w, n(t)) \neq 0$ for all $t, 0 \leq t \leq 1$, and that if $(w, \bar{n})=0$, then $(w, n(t))=0$ for all $t$. Therefore by setting $\varphi(\bar{p})=$ $(w, \bar{n}), \sum_{\alpha, \beta=1}^{n} A_{\alpha \beta} \lambda^{\alpha} \lambda^{\beta}$ is positive definite if $\varphi>0$, negative definite if $\varphi<0$, and identically 0 if $\varphi=0$, since $g^{\alpha \beta}(t)$ is positive definite for every $t$.

Now let $\bar{p}_{0}$ be a maximum point of $f$, so that $f(\bar{p}) \leq f\left(\bar{p}_{0}\right)$ for all $\bar{p}$ in $\bar{F}^{n}$. Such a point exists, since $\bar{F}^{n}$ is supposed to be compact. Then either $\varphi\left(\bar{p}_{0}\right) \neq 0$, or $\varphi\left(\bar{p}_{0}\right)=0$; the latter implies that $(w, n(t))=0$ for all $t$, and

$$
B_{\beta}=2 \int_{0}^{1}\left(g^{\alpha \beta} \frac{\partial(w, n)}{\partial u^{\alpha}}\right) d t .
$$

Since $(w, n(t)) \neq 0$, if $(w, \bar{n}) \neq 0$, then the set of points $\bar{p}$ on $\bar{F}^{n}$ where $(w, n(t))=0$ is the same as the set where $(w, \bar{n})=0$. Furthermore, if $(w, \bar{n})$ $>0$, then $(w, n(t))>0$, and

$$
\operatorname{grad}(w, n(t))=c(t) \operatorname{grad}(w, \bar{n}),
$$

with $c(t) \geq 0$. Thus

$$
\begin{aligned}
\sum_{\beta=1}^{n} \frac{\partial \varphi}{\partial u^{\beta}}\left(\bar{p}_{0}\right) & =2 \int_{0}^{1} g^{\alpha \beta} \frac{\partial(w, n(t))}{\partial u^{\alpha}} \frac{\partial(w, \bar{n})}{\partial u^{\beta}} d t \\
& =2 \int_{0}^{1} c(t) g^{\alpha \beta} \frac{\partial(w, \bar{n})}{\partial u^{\alpha}} \frac{\partial(w, \bar{n})}{\partial u^{\beta}} d t>0
\end{aligned}
$$

since $g^{\alpha \beta}$ is positive definite and $c(t) \geq 0, c(1)=1$. Therefore by our lemma, $f(\bar{p})=f\left(\bar{p}_{0}\right)$ in a neighborhood of $\bar{p}_{0}$; in other words, the set $U_{1}=\left\{\bar{p} \in \bar{F}^{n} \mid f(\bar{p})\right.$ $\left.=f\left(\bar{p}_{0}\right)\right\}$ is open in $\bar{F}^{n}$. This implies that $\bar{F}^{n}=U_{1} \cup U_{2}$, where $U_{2}=\{\bar{p} \epsilon$ $\left.\bar{F}^{n} \mid f(\bar{p})<f\left(\bar{p}_{0}\right)\right\}$, so that $\bar{F}^{n}$ is the disjoint union of two open sets. Since $\bar{F}^{n}$ is connected, it follows that $U_{1}=\bar{F}^{n}$, i.e., $f(\bar{p})=$ const. on $\bar{F}^{n}$. Hence the theorem is proved. 


\section{References}

[1] A. Aeppli, Einige Ähnlichkeits-und Symmetriesätze für differenzierbare Flächen im Raum, Comment. Math. Helv. 33 (1959) 174-195.

[2] H. Bruehlmann, Einige Kongruenzsätze für geschlossene k-dimensionale Flächen in $\mathrm{n}$-dimensionalen Riemannschen Räumen, Comment. Math. Helv. 44 (1969) 164-190.

[ 3 ] P. Hartman, Remarks on a uniqueness theorem for closed surfaces, Math. Ann. 133 (1957) 426-430.

[4] P. Hartman \& R. Sacksteder, On maximum principles for non-hyperbolic partial differential operators, Rend. Circ. Mat. Palermo (2) 6 (1957) 218-233.

[5] E. Hopf, Elementare Bemerkungen über die Lösungen partieller Differentialgleichungen zweiter Ordnung vom elliptischen Typus, S.-B. Preuss. Akad. Wiss. Berlin 19 (1927) 147-152.

[6] - - A remark on linear elliptic differential equations of second order, Proc. Amer. Math. Soc. 3 (1952) 791-793.

[7] H. Hopf \& Y. Katsurada, Some congruence theorem for closed hypersurfaces in Riemann spaces. II, Comment. Math. Helv. 43 (1968) 217-223.

[ 8 ] H. Hopf \& K. Voss, Ein Satz aus der Flächentheorie im Grossen, Arch. Math. 3 (1952) 187-192.

[9] Y. Katsurada, Some congruence theorems for closed hypersurfaces in Riemann spaces. I, Comment. Math. Helv. 43 (1968) 176-194.

[10] K. Voss, Einige differentialgeometrische Kongruenzsätze für geschlossene Flächen und Hyperflächen, Math. Ann. 131 (1956) 180-218.

UNIVERSITY OF British COLUMBIA, VANCOUVER University OF DORTMUND, GermanY 